\title{
A Definição de Função: Operacionalizar para Articular e Articular para Compreender
}

\section{The Definition of Function: Operationalize to Articulate and Articulate to Understand}

\author{
Jerson Sandro Santos de Souza ${ }^{a}$; Leandro de Oliveira Souza ${ }^{b}$ \\ a Instituto de Ciências Exatas, Universidade Federal do Amazonas, Manaus, Brasil - jersoncobain@ gmail.com \\ b Faculdade de Ciências Integradas do Pontal, Universidade Federal de Uberlândia, Uberlândia, Brasil - olilean@ gmail.com
}

\author{
Palavras-chave: \\ Função. Registros de \\ representação. \\ Dificuldades de \\ aprendizagem.
}

Resumo: Para compreender um conceito matemático é necessário saber articular as representações dele. Nesse sentido, articular as representações do conceito de função não é apenas saber identificar se determinada tabela, diagrama ou gráfico representa uma função, implica a construção semântica desse conceito. Acreditamos que a articulação entre as várias representações pode ser gerenciada pela definição de função que o aprendiz adota. Não qualquer definição, mas uma que seja realmente utilizada e que alcance todas as representações, o que denominamos de operacionalização da definição de função. Neste artigo, nosso objetivo é tecer reflexões sobre como favorecer a operacionalização da definição de função por parte do aprendiz. Para tal, fizemos um levantamento bibliográfico com foco na análise do desenvolvimento histórico-epistemológico do conceito de função e uma discussão sobre as múltiplas representações desse conceito. Concluímos apresentando três categorias que acreditamos favorecer a operacionalização e que podem ser objeto de estudo em projetos futuros.

\section{Keywords:}

Function. Registers of representation. Learning difficulties.

\begin{abstract}
To understand a mathematical concept it is needed to knowing to articulate the multiple representations of these concept. Articulate the representations of the concept of function it is not just about knowing how to identify if a table, diagram or graphics represents a function, imply to building a semantic knowledge of the concept. We believe that the articulation between various representations could be managed by the definition of function that learners would adopt. Not any definition, but one that be used and reach all representations, what we call operationalization of the definition of function. In this paper, our aim is to reflect about how to favoring the operationalization of the definition of function by learners. For this, we made a bibliographical survey focusing on the analysis of the historical-epistemological development of the concept of function, and a discussion on the multiple representations of this concept. We conclude by presenting three categories that we believe would favor the operationalization of the definition of function and that could be object of study in future projects.
\end{abstract}




\section{Introdução}

É muito comum em disciplinas como Química, Física e Biologia que a resolução de um problema esteja condicionada ao entendimento da noção de dependência entre duas variáveis. Isso ocorre porque muitos dos fenômenos em estudo apresentam regularidades, ou seja, desde que as condições iniciais sejam as mesmas eles possuem comportamento idêntico.

A regularidade de um fenômeno natural, no âmbito da sala de aula, é apresentada mediante uma regra de cunho causal que transcende as observações, geralmente representada por uma fórmula. Essa representação da lei de variação auxilia o professor na explicação do fenômeno, mas se o aprendiz não compreender as características e propriedades inerentes ao conceito matemático que dá vida à generalização apresentada, o processo de ensino e aprendizagem do fenômeno fica prejudicado. Essa é uma das dificuldades que a não compreensão do conceito de função pode acarretar.

O conceito de função é uma ferramenta própria para o estudo de leis quantitativas. Funções representam relações de dependência entre grandezas e o seu uso faz-se indispensável na leitura matemática de fenômenos da natureza. Esse fato amplia as possibilidades do conceito, não o restringindo a temas próprios da Matemática.

Existem várias maneiras de representar o conceito de função: palavras, diagramas, tabelas, fórmulas e gráficos. Na matemática escolar, outros conceitos foram estabelecidos como pré-requisitos para a apresentação da definição formal de função: estudo de noções de conjuntos e suas propriedades, conjuntos numéricos com ênfase no conjunto dos números reais, e o entendimento das noções de par ordenado e de produto cartesiano. Na sequência, o conceito de função é apresentado por meio de sua interpretação mais abstrata, geral e formal: como uma relação de dependência entre elementos de conjuntos quaisquer.

Apresentar funções dessa forma torna o conceito quase ininteligível. Vários termos girando em torno de um único conceito, várias representações e uma maneira estática de abordá-lo. Todo esse contexto pode desfavorecer a construção de significados claros, precisos e transferíveis.

Alguns estudos (VINNER, 1983; SIERPINSKA, 1992; DUVAL, 2012) apontam vários fatores que podem dificultar a aquisição do conceito de função, mas três desses fatores podem ser considerados cruciais: 1) a abordagem estática: quando o ensino do conceito privilegia aspectos estruturais e pouca ênfase é dada às suas aplicações; 2) ênfase quase exclusiva na representação analítica: um trabalho intensivo com fórmulas é realizado, ao ponto de o aprendiz conceber que função é uma fórmula e; 3) acréscimo de terminologias novas para identificar cada representação: dentro de cada representação criam-se macetes para saber identificar se uma tabela, gráfico ou diagrama representam o conceito de função, como se cada representação tivesse sua própria definição de função. 
Esses fatores podem aparecer isolados, mas é mais provável que os três permeiem todo o processo de ensino e aprendizagem do conceito de função. Mas por que geram dificuldades?

Segundo Duval (2012), a compreensão de um conteúdo conceitual em Matemática assume a coordenação de pelo menos dois registros de representação semiótica (termo usado para indicar diferentes tipos de representação, como escrita em língua natural, escrita algébrica, tabelas, figuras, etc). Quer dizer, é a articulação dos registros que constitui uma condição de acesso à compreensão Matemática, e não o isolamento em cada registro. Por isso acreditamos que os três fatores são tão prejudiciais, pois eles nutrem o isolamento dos registros de representação.

Como a compreensão do conceito de função pressupõe que o aluno transite, com rapidez e espontaneidade, entre as múltiplas representações desse conceito, possibilitar o processo de articulação das representações torna-se fundamental. Para tal, resolvemos focar num aspecto elementar de um conceito, a sua definição.

Acreditamos que o que gerencia a articulação entre as várias representações do conceito de função é a definição dele. Uma concepção de definição que alcance todas as representações. Em outras palavras, consideramos que a articulação é favorecida pelo processo de utilização consciente da definição como elo de ligação entre as demais representações, processo que denominamos de operacionalização da definição de função.

A operacionalização da definição de função é o momento em que o aprendiz apresenta uma definição formal ou pessoal (compatível com a formal) que abarque as várias representações, percebendo-as, por meio da definição adotada, como diferentes fontes de informações sobre o mesmo conceito. É o ponto em que o aprendiz trabalha com a definição que enunciou, ou seja, sabe definir o conceito de função e identificar/explicar, baseando-se na definição dada, se determinada tabela, diagrama ou gráfico representa uma função, além de conseguir identificar relações funcionais entre variáveis.

Em síntese, dentro da perspectiva apresentada, devemos primeiro operacionalizar a definição de função, depois articular as várias representações desse conceito, para, consequentemente, compreendê-lo. Como acreditamos que a operacionalização desencadeia o processo de aquisição do conceito de função, nossa pesquisa objetivou tecer reflexões sobre como favorecer a operacionalização da definição de função por parte do aprendiz. Para tanto, fizemos um levantamento bibliográfico com foco na análise do desenvolvimento históricoepistemológico do conceito de função, e uma discussão sobre as múltiplas representações desse conceito, discussão essa que atentou para a questão do acréscimo de terminologias novas toda vez em que uma representação é trabalhada. 


\section{Metodologia}

Optou-se pela realização de uma pesquisa bibliográfica para, por meio da leitura de diversos textos, buscar convergências a fim de sintetizar e discutir três variáveis cuja manipulação em situações de ensino favoreçam a operacionalização da definição de função. A pesquisa bibliográfica trata-se de uma modalidade de estudo e análise de documentos decorrentes de fontes científicas como livros, monografias, dissertações, teses e artigos científicos que, segundo Gil (2008, p.50), permite "ao investigador a cobertura de uma gama de fenômenos muito mais ampla do que aquela que poderia pesquisar diretamente". A pesquisa bibliográfica é desenvolvida a partir de material já elaborado, ou seja, pauta-se na contribuição de diferentes autores sobre o tema, então remete-se a fontes secundárias, pois os documentos já receberam tratamento analítico, entretanto, como aponta Marconi e Lakatos (2003, p.183), “a pesquisa bibliográfica não é mera repetição do que já foi dito ou escrito sobre certo assunto, mas propicia o exame de um tema sob novo enfoque ou abordagem, chegando a conclusões inovadoras". Em suma, “a pesquisa bibliográfica implica um conjunto ordenado de procedimentos de busca por soluções, atento ao objeto de estudo, e que, por isso, não pode ser aleatório" (LIMA; MIOTO, 2007, p. 38). Considerando os pressupostos mencionados, realizamos inicialmente a seleção e análise de textos científicos que apresentassem discussões de natureza psicológica, histórico-epistemológica e didática sobre o conceito de função.

\section{O desenvolvimento da definição do conceito de função}

Os problemas práticos ou teóricos são de extrema importância para o surgimento e desenvolvimento dos conceitos matemáticos, fato que tem o conceito de função como um grande exemplo. O desenvolvimento histórico desse conceito exemplifica como os consensos e discordâncias entre os pensadores, bem como os problemas que entravam em discussão dentro do meio matemático exigiam que as definições fossem cada vez mais precisas, que alcançassem todos os casos e que realmente exprimissem a essência do objeto matemático em questão. Quando as definições vigentes se mostravam insuficientes para a resolução dos novos problemas que iam aparecendo, os matemáticos se esforçavam para alcançar cada vez mais clareza, precisão e generalização.

Ponte (1990) relata que o surgimento da noção de função como conceito específico, objeto de estudo da Matemática, remonta apenas aos finais do século XVII, confundindo-se com os primórdios do Cálculo Infinitesimal. Este autor destaca que não se trata de uma noção muito antiga, conquanto aspectos muito simples desse conceito possam ser encontrados em épocas anteriores, presentes, por exemplo, na mais elementar operação de contagem. 
A formação do primitivo conceito de função era necessária devido aos problemas concretos do mundo físico que iam surgindo, associados à ideia de regularidade. Entretanto, outros elementos eram essenciais para a formação desse conceito, como a moderna notação algébrica, introduzida por Viète (1540-1603), e a representação geométrica, que proporcionava uma base intuitiva fundamental, possível devido à criação da Geometria Analítica por Descartes (1596-1650) e Fermat (1601-1665).

De forma vaga a ideia de função surgia em um trabalho de Newton (1642-1727) publicado em 1736, sob o título The Method of Fluxions and Infinite Series. Embora Newton não tenha usado a palavra função, percebe-se pelos seus trabalhos que ele considerava as relações de dependência entre variáveis. Usava o termo relata quantitas para designar o que chamamos hoje de variável dependente, fluente para designar as variáveis independentes e genita para indicar as quantidades obtidas de outras a partir das quatro operações aritméticas fundamentais.

Foi Leibniz (1646-1716) quem pela primeira utilizou o termo função, em 1673. Ele usou a palavra função para indicar, em termos muito gerais, quantidades que variavam ao longo de uma curva (e.g. tangente). Ele também introduziu os termos parâmetro, constante e variável. "Leibniz não é o responsável pela moderna notação para função, mas é a ele que se deve a palavra "função", praticamente no mesmo sentido em que é usada hoje" (BOYER, 1996, p.297).

Os problemas que deram origem ao Cálculo (desenvolvido por Newton e Leibniz) eram geométricos e cinemáticos, por isso, o foco do Cálculo, até então, era o estudo das curvas geométricas, não do conceito de função em si. Contudo, o procedimento de relacionar curvas a fórmulas viria mudar esse quadro.

Com o desenvolvimento do estudo de curvas por meios algébricos, tornou-se indispensável um termo para representar quantidades dependentes de alguma variável por meio duma expressão analítica. Com esse propósito, a palavra "função" foi adotada na correspondência trocada entre 1694 e 1698 por Leibniz e João Bernoulli (PONTE, 1990, p. 3).

Em 1718, Johann Bernoulli (1667-1748), num primeiro intento de deixar mais preciso o conceito de função, define função de uma grandeza variável, segundo Youschkevitch (1976), a uma quantidade composta, de um modo qualquer, desta grandeza variável e de constantes. Para Bernoulli, uma função só poderia ser representada por uma única expressão analítica. Ele empreendeu várias notações para uma função, sendo fx a que mais se aproxima da atual.

A definição de Bernoulli seria aperfeiçoada em 1748 por Euler (1707-1783), seu antigo aluno. Em sua obra Introductio in Analysin Infinitorum, Euler, após definir quantidade constante e quantidade variável, afirmava, segundo Youschkevitch (1976), que uma função de uma quantidade variável é uma expressão analítica composta, de um modo qualquer, desta 
quantidade e de números ou quantidades constantes. Euler não definiu o que seria uma expressão analítica, mas, segundo Boyer (1996), ele se referia às funções algébricas e às funções transcendentes elementares (exponenciais, logarítmicas e trigonométricas). Embora Euler não tenha sido o precursor no que se refere à noção de função, foi ele o primeiro a tratar o Cálculo como uma teoria formal de funções, sua obra de 1748 foi a primeira em que o conceito de função desempenhou um papel central. Assim como Leibniz, Euler foi um fecundo criador de notações, deve-se a ele a notação $f(x)$ até hoje utilizada para designar uma função de $x$.

As ideias de Bernoulli e Euler iriam revolucionar o estudo das funções. A partir de então, pensar em função seria o mesmo que pensar na expressão analítica que a representava, "situação que haveria de vigorar pelos séculos XVIII e XIX, apesar de cedo se perceber que conduzia a diversas incoerências e limitações (de fato, uma mesma função pode ser representada por diversas expressões analíticas!)" (PONTE, 1990, p. 4).

A observação de fenômenos físicos levou os matemáticos, no decurso da história, a ampliarem a compreensão dos objetos matemáticos e definirem esses objetos com maior precisão. Podemos destacar dois problemas de extrema importância para a evolução do conceito de função: o problema da corda vibrante e o problema da condução do calor. Esses problemas, que desafiaram os matemáticos daquela época, evidenciaram as limitações do conceito de função, situação que implicaria sucessivas ampliações do mesmo, alterando profundamente a sua natureza e o seu significado.

O problema da corda vibrante ocasionou discussões mais acaloradas acerca do conceito de função, o que culminaria no enfraquecimento da quase inseparável relação função-expressão analítica. Segundo Ávila (1985, p. 16), “O problema surgiu em meados do século XVIII, em conexão com o estudo das vibrações transversais de uma corda flexível e esticada, como uma corda de violino".

Simplificadamente (abstraindo algumas suposições iniciais), o problema consistia, segundo Botelho e Rezende (2007), no seguinte: considere uma corda esticada e com os extremos fixos (corda de algum instrumento musical, como o violão). A corda será afastada de sua posição de repouso (figura 1), assumindo a posição inicial (figura 2), e depois solta, provocando vibrações. Sendo assim, descreva matematicamente os movimentos dessa corda, ou seja, determine a função que descreve o formato da corda quando variamos a posição x e o tempo $t$. 


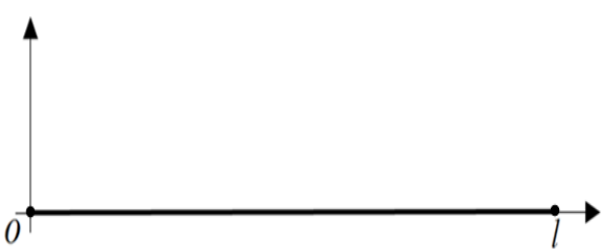

Figura 1 - Posição de repouso Fonte: Os autores.

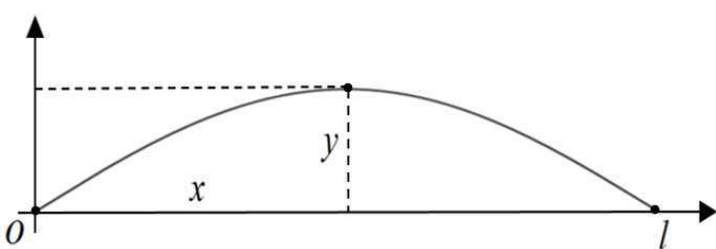

Figura 2 - Posição inicial Fonte: Os autores.

O problema era desafiador para os matemáticos da época, até porque tanto os conceitos quanto os procedimentos que eles usavam eram imprecisos. Ávila (1985, p.19) afirma que "não só o conceito corrente de função era restrito e impreciso, como não existia uma fundamentação adequada das noções de limite, derivada e integral, ou uma teoria de convergência de séries".

Como resposta ao problema da corda vibrante, d'Alembert (1717-1783), o primeiro a estudar significativamente o fenômeno das vibrações de uma corda, apresentou em 1747, no seu artigo intitulado Recherches sur la courbe que forme une corde tendue mise en vibration, a seguinte equação que ficaria conhecida como equação da onda (ou equação das cordas vibrantes), em terminologia moderna:

$$
\frac{\partial^{2} y}{\partial x^{2}}(x, t)=\frac{\mu}{T} \frac{\partial^{2} y}{\partial t^{2}}(x, t)
$$

onde $y=y(x, t)$ representa a amplitude de um ponto de abscissa $x$ no instante $t, \mu$ é a densidade linear de massa da corda e $T$ é a força de tensão na corda.

Para a equação (1), geralmente apresentada na forma $\frac{\partial^{2} y}{\partial x^{2}}(x, t)=\frac{1}{v^{2}} \frac{\partial^{2} y}{\partial t^{2}}(x, t)$, d'Alembert obteve a seguinte solução geral: $y(x, t)=f(x+v t)+g(x-v t)$, onde $f$ e $g$ são funções arbitrárias determinadas pela posição inicial da corda e $v$ é uma constante que depende da situação física estudada.

Para d'Alembert, a curva que representa a corda em sua posição inicial deveria ser descrita por uma expressão analítica. Embora tanto Daniel Bernoulli (1700-1782) quanto Euler concordassem com a solução de d'Alembert, Bernoulli defendia que não era preciso restringir a curva que representava a corda em sua posição inicial a uma expressão analítica (Euler também ponderava sobre essa restrição).

Daniel Bernoulli, adotando um ponto de vista bastante físico, fez uma observação profunda, retomada anos depois por Fourier (1768-1830). Ele acreditava que a posição inicial da corda poderia ser descrita como uma soma possivelmente infinita de termos trigonométricos. Ele apresentou uma solução em série trigonométrica para a equação (1), mas não foi muito bem aceita. 
Lagrange (1736-1813) obteve uma solução da equação (1) na forma de uma série mais geral que a solução obtida por Daniel Bernoulli, inclusive foi ele quem mais se aproximou do desenvolvimento de uma dada função em série trigonométrica.

O debate entre envolvendo Euler, d'Alembert, Daniel Bernoulli e Lagrange perdurou por alguns anos, mostrando-se frutífero para a expansão do conhecimento funcional. Segundo Kliner (1989), esses diálogos tiveram importantes consequências na evolução do conceito de função. Este autor afirma que o conceito foi estendido, de modo a abranger: a) funções definidas por expressões analíticas diferentes em diferentes intervalos e; b) funções desenhadas à mão livre e que, possivelmente, não eram dadas por combinações de símbolos algébricos.

Fourier, ao estudar o problema de condução do calor nos objetos materiais, teve que retomar as ideias sugeridas no problema da corda vibrante. Ele conjecturou, a certa altura, que "qualquer" função poderia ser escrita como uma soma de senos e cossenos, num intervalo apropriado. Embora não tenha dado uma prova matemática dessa afirmação, instigou outros matemáticos, como Dirichlet (1805-1859) e Cantor (1845-1918), a definirem o real alcance do dito "qualquer".

Em sua obra Théorie analytique de la chaleur, publicada em 1822, Fourier afirma que certas funções $y=f(x)$ podiam ser representadas por uma série da forma:

$$
\begin{aligned}
& f(x)=\frac{a_{0}}{2}+\sum_{n=1}^{\infty}\left(a_{n} \cdot \cos (n x)+b_{n} \cdot \operatorname{sen}(n x)\right), \text { onde } a_{0}, a_{n} \text { e } b_{n} \text { são dados por } \\
& a_{0}=\frac{1}{\pi} \cdot \int_{-\pi}^{\pi} f(x) d x, a_{n}=\frac{1}{\pi} \cdot \int_{-\pi}^{\pi} f(x) \cos (n x) d x, n \geq 0 \text { e } b_{n}=\frac{1}{\pi} \cdot \int_{-\pi}^{\pi} f(x) \operatorname{sen}(n x) d x, n \geq 1 .
\end{aligned}
$$

Os coeficientes $a_{0}, a_{n}$ e $b_{n}$ são os chamados coeficientes de Fourier da função $f$. Com esses coeficientes, uma série da forma (2) é chamada a série de Fourier.

A relação (2) conduziu Fourier, naturalmente, ao estudo de condições de integrabilidade. Segundo Ávila (1985), Fourier apresentou pela primeira vez, num artigo de 1807, submetido à Academia de Ciências da França, a questão: dada a função $f$, é sempre possível achar esses coeficientes $\left(a_{0}, a_{n}\right.$ e $\left.b_{n}\right)$ de modo a satisfazer a relação (2)? Este autor afirma que essa questão surgira, ainda que numa situação bem restrita, com Daniel Bernoulli em 1753, entretanto foi com Fourier que ela se tornou realmente presente no mundo matemático.

Quanto aos tipos de funções que podem ser estudadas, a representação de funções por Séries de Fourier fornece uma generalidade que supera o desenvolvimento por Séries de Taylor. De fato, Fourier mostrou que muitas funções poderiam ser representadas por uma Série de Fourier. Mesmo que existam muitos pontos em que a derivada não exista ou em que 
a função não seja contínua, a função pode ter expansão em série de Fourier (BOYER, 1996). Assim, as ideias defendidas por Fourier provocaram uma espécie de movimento de libertação do conceito de função de sua representação analítica.

Dirichlet se empenhou para entender em quais casos as integrais que definem os coeficientes de uma série de Fourier existiam, por isso foi levado a debruçar-se sobre o conceito de função. Conforme Ponte (1990, p. 4), ele

formulou as condições suficientes para representabilidade duma função por uma série de Fourier. Dirichlet separou então o conceito de função da sua representação analítica, formulando-o em termos de correspondência arbitrária entre conjuntos numéricos, em 1837.

A definição de Dirichlet acabou sendo um marco para a concepção moderna do conceito de função. Na segunda metade do século XIX, uma onda de exemplos de funções baseada na definição de Dirichlet foi apresentada pelos matemáticos daquele período (COSTA, 2004).

Dirichlet definiu função, segundo Rüthing (1984), da seguinte maneira:

Suponhamos que a e b são dois valores dados e $\mathrm{x}$ é a quantidade variável que assume, gradualmente, todos os valores localizados entre a e b. Se para cada $\mathrm{x}$ corresponde um único $\mathrm{y}$, de modo que, enquanto $\mathrm{x}$ percorre o intervalo de a até $\mathrm{b}$, $y=f(x)$ varia gradualmente da mesma forma, então y é chamada função contínua de x para este intervalo. Além disso, não é absolutamente necessário que y dependa de x no intervalo inteiro de acordo com a mesma lei; sem dúvida, não é necessário pensar somente em relações que possam ser expressas através de operações matemáticas.

Para mostrar a natureza totalmente arbitrária da lei de correspondência que orienta a relação entre as variáveis, Dirichlet surpreendeu os matemáticos ao apresentar, em 1829, o exemplo de uma função que era descontínua em todos os seus pontos, não integrável e não podia ser representada por uma expressão analítica (pelo menos na época). Função hoje conhecida como Função de Dirichlet. Abaixo temos a definição da função de Dirichlet, a função $d$.

$$
d: \mathbb{R} \rightarrow \mathbb{R} \text { definida por } d(x)=\left\{\begin{array}{l}
1, \text { se } x \in \mathbb{Q} \\
0, \text { se } x \in(\mathbb{R}-\mathbb{Q})
\end{array}\right.
$$

A definição de Dirichlet está mais próxima do ponto de vista atual de uma correspondência entre dois conjuntos de números, mas os conceitos de conjunto e de número real não tinham ainda sido estabelecidos (BOYER, 1996). Contudo, foi a partir dos estudos de conjuntos de pontos feito por Cantor e, consequentemente, do desenvolvimento da Teoria dos Conjuntos, que o conceito de função passaria a ser definido em termos de pares ordenados de elementos, não necessariamente numéricos (FONSECA et al., 2013).

Cantor, também motivado pelo estudo das séries de Fourier, tinha o objetivo de saber em que condições uma série de Fourier convergia para a função a que estava associada. Ao 
longo de seus estudos percebeu que para obter uma resposta precisa a essa pergunta tinha que focar não somente o conceito de função, mas também o conceito de número; portanto, o que seria um número real? Essa nova questão o conduziu ao estudo dos subconjuntos da reta, o que ocasionou nos estudos iniciais da Teoria dos Conjuntos.

Foi, finalmente, com a definição de um grupo de matemáticos franceses, que o conceito de função atingiu o seu caráter mais geral e formal. O referido grupo ficou conhecido como Nicolas Bourbaki.

Já no século XX, a busca pela formalização dos conceitos matemáticos levou muitos pesquisadores matemáticos a publicarem textos científicos. Entre eles destaca-se um grupo de matemáticos da França, que adotou o pseudônimo de Nicolas Bourbaki. Esse grupo acreditava que muitas definições da matemática moderna deveriam ser repensadas. Para isso, escreveram uma série de livros, que foram publicados a partir de 1935, apresentando ao mundo "a matemática moderna", a partir de uma nova terminologia e novos conceitos (FONSECA et al., 2013, p. 12).

A definição de Bourbaki, que até hoje influencia o ensino de funções, foi importante para ampliar as possibilidades do conceito. A definição dada pelo grupo, em 1939, conforme Rüthing (1984), pode assim ser enunciada:

Sejam E e F dois conjuntos distintos ou não. Uma relação entre uma variável x de E e uma variável y de $\mathrm{F}$ é dita uma relação funcional em $\mathrm{y}$, ou relação funcional de $\mathrm{E}$ em $\mathrm{F}$, se, para qualquer $\mathrm{x} \in \mathrm{E}$ existe um único $\mathrm{y} \in \mathrm{F}$, e apenas um, que está na relação dada com x. Damos o nome de função à operação que associa a todo elemento $\mathrm{x} \in \mathrm{E}$ o elemento $\mathrm{y} \in \mathrm{F}$ que se encontra na relação dada com $\mathrm{x}$; dizemos que y é o valor da função para o elemento $x$, e que a função é determinada pela relação funcional considerada. Duas relações funcionais equivalentes determinam a mesma função.

Podemos observar que a definição de Bourbaki apresenta o conceito de função como um certo subconjunto do produto cartesiano $E x F$. Assim, com essa definição de caráter mais abrangente, a noção de função se expandiu de forma a incluir tudo o que fossem correspondências arbitrárias entre quaisquer conjuntos, e não apenas numéricos. Segundo Alvarenga et al. (2014, p. 175), "Esta característica foi essencial para a disseminação do conceito de função por diversas áreas do conhecimento, podendo, assim, ser utilizada em distintos modelos de correspondência entre variáveis, não sendo restrita apenas ao conjunto dos números reais".

De resto, podemos dizer que o conceito de função passou por diversas transformações necessárias para torná-lo cada vez mais fundamentado, cada vez mais útil para a resolução de problemas, cada vez mais operacional. Segundo Silva e Rezende (1999), através da análise histórica do conceito de função, podemos perceber que os matemáticos, ao definirem esse conceito, utilizaram três ideias básicas:

a) função como relação entre quantidades variáveis: Essa interpretação elege a noção de variação como o cerne do conceito de função. Ela se refere à ideia de duas grandezas que variam uma dependendo da outra, ideia esta tão presente no nascimento da Física quantitativa 
e que permeia nosso cotidiano. Tal interpretação mostra, de imediato, a utilidade prática do conceito de função.

b) função como relação entre conjuntos: É a ideia de que a cada elemento x de um conjunto A se associa um único elemento $\mathrm{f}(\mathrm{x})$ de um conjunto B. Embora a interpretação anterior seja a mais intuitiva, a relação entre conjuntos é a ideia mais utilizada para definir o conceito de função. Esta interpretação é estática e tem um caráter mais formal que as demais.

c) função como transformação: Interpretação dada pelo matemático George Boole (1815-1864) em sua definição. É a ideia de que a função f transforma o elemento x no valor $f(x)$. Esta se faz presente, por exemplo, nas transformações lineares entre espaços vetoriais ou nas funções complexas de variáveis complexas.

O percurso histórico do conceito de função deixa claro o sentido de sua evolução e operacionalização: de ideias mais intuitivas a ideias mais gerais e formais, em outras palavras, da relação entre quantidades variáveis à relação entre conjuntos.

A presente análise histórico-epistemológica nos possibilitou entender que a construção de um conceito depende de tantas outras ideias e de uma gestação lenta em que os problemas e o instrumento matemático se inter-relacionam, ajudando-se e esclarecendo-se mutuamente. Percebemos, ainda, que o conceito de função surgiu dentro de um contexto prático, atrelado a problemas com referência na realidade, como uma relação entre quantidades variáveis, evidenciando um apelo fortemente intuitivo e rico para o entendimento de suas limitações enquanto objeto matemático. Conforme novos problemas iam aparecendo, as limitações das definições então adotadas eram explicitadas, o que exigia mais clareza, precisão e generalização. Esse processo de constante aperfeiçoamento culminou na definição de função como relação entre conjuntos quaisquer, a mais formal e mais utilizada para introduzir o tema.

Obedecendo ao sentido histórico de evolução do conceito de função, acreditamos que podemos favorecer a operacionalização na medida em que o conceito seja apresentado como relação entre quantidades variáveis para depois ser formalizado como relação entre conjuntos. Situações mais intuitivas, principalmente aquelas que aludem ao universo mais próximo do aluno, são fundamentais para que o aprendiz atribua significação ao objeto matemático em questão, o que possibilitaria ao mesmo trabalhar com a definição que adotou. Conforme Silva e Rezende (1999, p.32), "se a interpretação como relação entre quantidades variáveis torna a aprendizagem do conceito de função mais intuitiva para o aluno e de maior aplicabilidade nas demais áreas do conhecimento, por que não partir dela para, após o domínio do conceito, chegar ao formalismo?"

Partindo dessa mesma preocupação, Botelho e Rezende (2007, p. 74) afirmam:

Não pensamos em fórmulas matemáticas ou em subconjuntos de um produto cartesiano quando compramos um produto. O que fazemos é relacionar a quantidade comprada com o preço a ser pago através do conhecimento que temos sobre a maneira com que estas grandezas, quantidade e preço, variam. 


\section{As múltiplas representações do conceito de função}

Entendemos que para favorecer a operacionalização também é necessário inserir o conceito de função em diferentes contextos problemáticos; contextos baseados na pluralidade de representações. Por isso, nesta seção, apresentaremos alguns exemplos em que discutimos os contras e prós de cada representação e sobre o principal fator que causa o isolamento das representações: o acréscimo desnecessário de terminologias novas.

Exemplo 1: Observe a seguinte sequência de figuras:

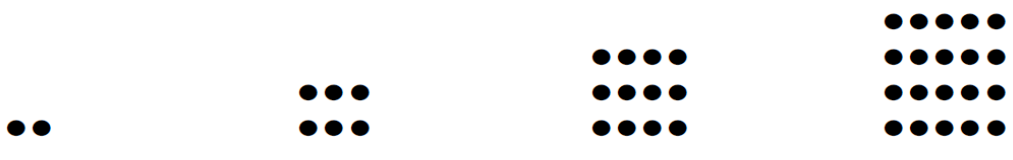

Figura 3 - Sequência dos números retangulares.

Fonte: Os autores.

Se as próximas figuras da sequência obedecem ao mesmo padrão observado nas figuras iniciais, então quantas bolinhas terá a centésima figura?

É claro que, com muito esforço, essa questão pode ser resolvida sem fazer apelo a um raciocínio mais elaborado. Basta desenhar mais 96 figuras e o problema estará resolvido. Contudo, o dito raciocínio mais elaborado pode tanto reduzir o trabalho quanto facilitar o entendimento do comportamento do "fenômeno" como um todo. Isto é possível, em particular, pois o nosso fenômeno fictício possui uma regularidade.

A tal regularidade pode ser percebida pela forma como os "pontinhos" são dispostos, de modo a formarem retângulos. Partindo desse fato, e sem recorrer ao trabalho árduo de desenhar várias figuras, como poderíamos resolver o problema?

Se nos basearmos nas limitações de ação, teremos de saber calcular a quantidade de pontinhos de uma figura conhecendo apenas o número natural que representa sua posição. Podemos facilitar o entendimento do problema recorrendo à representação tabular.

Representação Tabular

Primeiro poderíamos construir uma tabela com duas linhas, a primeira será a linha das posições e a segunda a da quantidade de pontinhos, observe a tabela 1.

Tabela 1 - Dados organizados de forma a dar uma noção inicial da lei quantitativa

\begin{tabular}{|c|c|c|c|c|c|c|c|c|}
\hline Posição & 1 & 2 & 3 & 4 & 5 & 6 & 7 & 8 \\
\hline Quantidade & 2 & 6 & 12 & 20 & 30 & 42 & 56 & 72 \\
\hline
\end{tabular}

Fonte: Os autores.

As posições 5, 6, 7 e 8 puderam ser preenchidas, pois um olhar mais cauteloso sobre as quatro primeiras posições sugere uma lei: a quantidade de pontinhos é o produto da posição atual pela posição seguinte. Essa afirmação pode ser validada se pensarmos na quantidade de pontinhos de certa posição como o resultado do cálculo da área do retângulo 
dessa posição. Podemos supor que a quantidade de pontinhos horizontais é a medida da base e a quantidade de pontinhos verticais é a medida da altura. Como a área de um retângulo é o produto da base pela altura, temos, por exemplo, o caso da figura 4.

\begin{tabular}{|cc|}
\hline Figura da $3^{\mathrm{a}}$ posição & Figura da $4^{\mathrm{a}}$ posição \\
$3 \times 4=12$ pontinhos & $4 \times 5=20$ pontinhos \\
$\frac{0}{4}$ & $\frac{0}{5}$ \\
\hline
\end{tabular}

Figura 4 - Quantidade de pontinhos das figuras das $3^{\mathrm{a}}$ e $4^{\mathrm{a}}$ posições.

Fonte: Os autores.

A tabela nos ajuda a organizar os dados e a empreender o tal raciocínio mais elaborado, contudo ainda estamos presos a casos particulares.

A tabela 1 dá uma ideia inicial da lei. Ela consiste em duas sucessões, dois conjuntos numéricos, o das posições $P$ e o dos números de pontinhos $\mathrm{Q}$, postos em correspondência um com o outro. Tal correspondência é unívoca no sentido de $P$ para $Q$, pois a cada posição corresponde uma, e apenas uma, quantidade de pontinhos. A lei está na forma como essa correspondência do conjunto $P$ ao conjunto $Q$ se realiza.

Assim, é natural entendermos que o instrumento para o estudo de leis quantitativas, o conceito de função, deve ter em sua essência a correspondência de dois conjuntos. De posse dessa ideia fundamental, o próximo passo seria generalizar. Para tal, devemos criar uma representação simbólica, afim de alcançar a generalidade conveniente, uma característica da lei, desprendendo-se de tabelas de resultados particulares.

Segundo Caraça (1951), a representação simbólica almejada e, consequentemente, a generalização, é alcançada a partir do momento em que se introduz o conceito de variável.

Seja (E) um conjunto qualquer de números, conjunto finito ou infinito, e convencionemos representar qualquer dos seus elementos por um símbolo, por ex.: x. A este símbolo, representativo de qualquer dos elementos do conjunto (E), chamamos variável [...] Quando dizemos, por exemplo: seja (E) o conjunto dos números reais do intervalo $(0,1)$, e seja $\mathrm{x}$ a sua variável, que queremos significar? Que o símbolo x sem coincidir individualmente com nenhum dos números reais desse intervalo, é suscetível de os representar a todos; é, afinal, o símbolo da vida coletiva do conjunto, vida essa que se nutre da vida individual de cada um dos seus membros, mas não se reduz a ela (CARAÇA, 1951, p. 127).

Estabelecido o conceito de variável, agora podemos voltar ao exemplo 1 e apresentar uma noção mais geral, cuja essência seja a correspondência entre o conjunto das posições $P$ e o conjunto das quantidades $Q$, a representação algébrica ou analítica.

Representação Analítica

Considere $p$ a variável do conjunto $P$ e $q$ a variável do conjunto $Q$. A lei consiste na existência duma dada correspondência entre $p$ e $q$, correspondência que sabemos ser unívoca no sentido $P \rightarrow Q$. Diremos que a variável $q$ é função da variável $p$, indicaremos 
simbolicamente por $q=f(p)$, onde $p$ será chamada de variável independente e q variável dependente. A variável $q$ é dependente da $p$, pois sua variação depende da variação dos valores de $p$, particularmente, variando a posição, variamos, também, a quantidade de pontinhos.

Agora é possível transcrever a lei: a quantidade de pontinhos é o produto da posição atual pela posição seguinte, para a linguagem algébrica:

$$
q=p \cdot(p+1) \text { ou } f(p)=p \cdot(p+1),
$$

onde $p$ é a posição da figura, $p+1$ é a posição seguinte e $q$ é a quantidade de pontinhos da figura de posição $p$.

De posse de (3), saber quantos pontos comporta a centésima posição fica simples, basta fazermos $p=100$, em (3):

$$
q=f(100)=100 .(100+1)=100.101=10100 \text { pontinhos } .
$$

Ao descobrirmos a lei quantitativa alcançamos um certo poder de previsão e controle sobre o fenômeno em estudo, por isso é tão útil interpretar o conceito de função como uma relação entre quantidades variáveis. Agora podemos saber, sem que precisemos recorrer a casos particulares, quantos pontinhos há em qualquer posição e mais, se for dada a quantidade de pontinhos de uma figura, podemos saber qual a posição que ela ocupa na sequência. Por exemplo, qual é a posição da figura que possui 20 pontinhos? Basta fazermos $q=20$ em (3) e resolver a equação resultante: $p \cdot(p+1)=20$.

A equação $p \cdot(p+1)=20$ pode ser escrita como $p^{2}+p=20$, subtraindo 20 em ambos os membros dessa equação chegamos à equação do segundo grau na forma completa: $p^{2}+p-20=0$, temos que $\Delta=1^{2}-4.1 .(-20)=81$, então $p=\frac{-1 \pm \sqrt{81}}{2}=\frac{-1 \pm 9}{2}$. Como delta é positivo a equação tem duas raízes reais e distintas como solução, são elas $p^{\prime}=\frac{-1+9}{2}=\frac{8}{2}=4$ e $p^{\prime \prime}=\frac{-1-9}{2}=\frac{-10}{2}=-5$. Como as posições são dadas por números naturais, a solução conveniente para o problema é 4, ou seja, a figura composta de 20 pontinhos está na quarta posição.

\section{Definição do conceito de função}

$\mathrm{Na}$ tabela 1 estão indicados apenas alguns pares de valores da correspondência, embora a tabela nos dê uma primeira noção, ela é limitada. Por outro lado, a afirmação $q=f(p)$ significa que qualquer valor $p$ corresponde um único valor de $q$. Dentro desse contexto mais abrangente, podemos definir o conceito de função da seguinte maneira: 
Dados os conjuntos X, Y, uma função $f: X \rightarrow Y$ (lê-se: "uma função de $\mathrm{X}$ em Y") é uma regra (ou conjunto de instruções) que diz como associar a cada elemento $x \in X$ um elemento $y=f(x) \in Y$. O conjunto X chama-se domínio e Y é o contradomínio da função f. Para cada $x \in X$, o elemento $f(x) \in Y$ chamase a imagem de $\mathrm{x}$ pela função $\mathrm{f}$, ou o valor assumido pela função $\mathrm{f}$ no ponto $x \in X$ (LIMA et al., 2012, p.45).

Vale ressaltar que o ensino de funções é quase exclusivamente centrado na apresentação de correspondências entre conjuntos numéricos, as chamadas funções numéricas. Embora esses tipos de funções sejam as mais importantes para o estudo de leis quantitativas, o conceito de função não se restringe a correspondências entre conjuntos numéricos. Muitos exemplos de funções são correspondências entre conjuntos numéricos, onde a regra que expressa a regularidade é dada por uma fórmula, "mas em geral não precisa ser assim. A natureza da regra que ensina como obter $f(x)$ quando é dado $x$ é inteiramente arbitrária" (LIMA et al., 2012, p. 49). Isso pode ser observado no exemplo a seguir.

Exemplo 2: Diante de uma coleção de objetos, se solicitado a contar os mesmos, um indivíduo quase que automaticamente procede da seguinte maneira: aponta para um dos objetos dizendo: um; aponta para outro e diz: dois; e assim procede até esgotar os objetos da coleção. Sendo, por exemplo, 5 o último número pronunciado, ele conclui que a coleção tem 5 objetos.

Portanto, a contagem se realiza fazendo corresponder, sucessivamente, a cada objeto da coleção um número da sequência de números naturais $(1,2,3, \ldots)$. A correspondência ou associação mental de dois entes exige que haja um ponto de partida e um ponto de chegada, respectivamente, o objeto a ser contado e o número natural que o rotula, e, ainda, uma ideia (ou conjunto de instruções) pela qual o pensar no ponto de partida evoca o pensar no ponto de chegada, a chamada lei de correspondência.

Pode-se dizer que uma função $f$ é um objeto matemático constituído por três partes:

1) Domínio A: conjunto de partida ou conjunto onde a função está definida.

2) Contradomínio B: conjunto de chegada ou conjunto onde a função toma valores.

3) Lei de correspondência $f(x)$ : é a maneira pela qual o pensar no elemento do domínio desperta o pensar no elemento do contradomínio. Quando a função é numérica, tanto o domínio quanto o contradomínio são conjuntos numéricos, nesse caso, a lei de correspondência será dada por uma expressão analítica.

A notação abaixo sintetiza em um resumido conjunto de símbolos a participação dos três elementos constituintes do conceito de função.

$$
\text { Notação: } \begin{aligned}
& f: A \rightarrow B \\
& x \propto \quad y=f(x)
\end{aligned}
$$


Essa notação indica uma função $f$, definida em $A$ com imagens (i.e., pontos de chegada) em $B$ segundo a lei de correspondência $y=f(x)$. A representação $A \rightarrow B$ indica a correspondência entre os conjuntos $A$ e $B$, enquanto $x \alpha \quad y$ indica a correspondência entre os elementos dos respectivos conjuntos. No caso das funções numéricas, a identidade $y=f(x)$ nos informa que os pontos de chegada $y$ são construídos mediante uma fórmula dependente de $x$. Sendo assim, o elemento $y$ é equiparado a forma genérica de calculá-lo $f(x)$.

A lei de correspondência que caracteriza uma função deve satisfazer duas condições, conforme Lima et al. (2012):

I) Sem exceções: a lei de correspondência deve fornecer pontos de chegada para todo ponto de partida, ou seja, cada elemento (todos) do domínio deve estar associado a algum elemento do contradomínio;

II) Sem ambiguidades: cada elemento do domínio está associado a apenas um elemento do contradomínio.

A partir dessa definição, podemos dizer que o exemplo 2 representa bem a ideia de função. Possui um domínio (coleção de objetos), um contradomínio (conjunto dos naturais) e uma lei de correspondência (a regra), que se configura no próprio processo elementar de contagem: a cada objeto da coleção apontado se atribui um número natural, como se fosse um rótulo, começando do número 1 e procedendo sequencialmente até findar os objetos do conjunto. A lei de correspondência obedece às duas condições: sem exceções e sem ambiguidades, pois cada objeto da coleção recebe algum rótulo e todos os objetos recebem um único rótulo, caso contrário, algum objeto ficaria fora da contagem ou seria computado mais de uma vez. No exemplo 1, os conjuntos de partida e chegada são, respectivamente, $P$ e $Q$ (ambos conjuntos de números naturais). Por ser uma função numérica admite como lei de correspondência uma fórmula (expressão analítica), no caso, $f(p)=p \cdot(p+1)$, que é a tradução algébrica da lei: a quantidade de pontinhos é o produto da posição atual pela posição seguinte. Essa lei de correspondência obedece às condições I e II pois cada posição apresenta alguma quantidade de pontinhos (que define a figura), e toda posição está associada a uma única quantidade de pontinhos (senão cada posição poderia ter mais de uma figura).

Representação por diagramas

Além de definições, tabelas e fórmulas, o conceito de função pode, também, ser representado por diagramas de flechas e por gráficos. O diagrama de flechas se configura numa tentativa de apresentar o conceito de função na sua forma mais geral e formal como relação entre conjuntos, já no ensino básico. O exemplo 2, em termos de diagramas, poderia ser representado assim: 


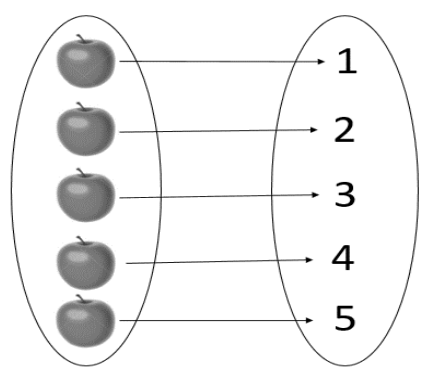

Figura 5 - Diagrama para a contagem de cinco maçãs.

Fonte: Os autores.

Com o auxílio do diagrama de flechas, duas condições devem ser satisfeitas para que uma relação $f$ de $A$ em $B$ seja considerada uma função:

i) todo elemento do conjunto $A$, domínio, deve servir como ponto de partida de flecha;

ii) cada elemento do conjunto $A$ deve servir como ponto de partida de uma única flecha.

É fácil ver que o diagrama da figura 5 representa uma função, pois cumpre as condições i e ii. As condições i e ii são apenas as condições sem exceção e sem ambiguidade que a lei de correspondência deve satisfazer para que uma relação entre dois conjuntos seja uma função. Ou seja, há o acréscimo desnecessário de terminologias novas. Para compreender melhor as condições i e ii, observe a figura 6.

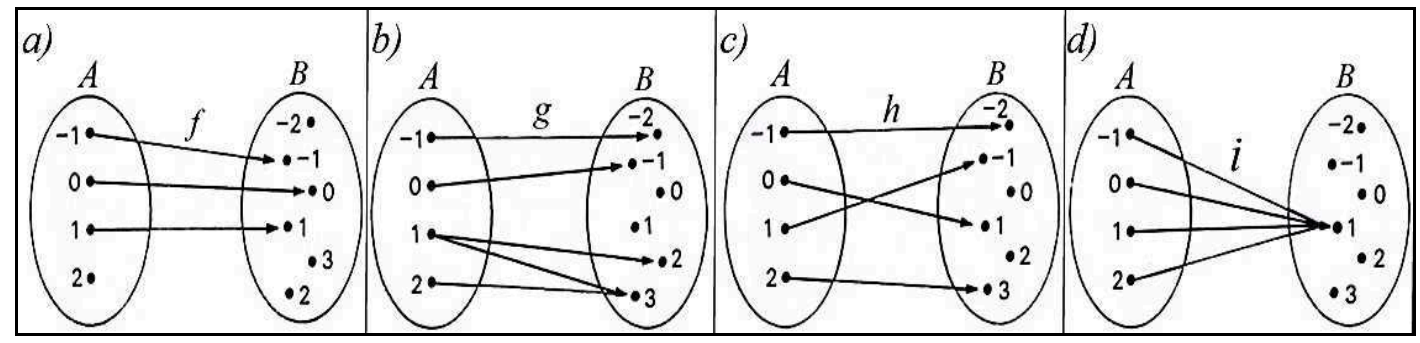

Figura 6 - Diagramas de flechas exemplificando relações entre conjuntos numéricos. Fonte: Os autores.

Os diagramas $c$ e $d$ representam o conceito de função. No diagrama $d$ acontece algo que pode confundir a aplicação das duas condições: um ponto de partida deve estar associado a um único ponto de chega, mas um ponto de chegada pode receber vários pontos de partida. O diagrama $a$ não representa uma função, pois não cumpre i (sem exceção); e o diagrama $b$, pois não cumpre ii (sem ambiguidades).

Geralmente, no que diz respeito ao ensino de funções, a representação por diagramas é a mais utilizada, principalmente quando se inicia o aprendiz nesse conceito. O que acontece é que o ensino de funções vai se resumir a dizer se um diagrama representa ou não o conceito de função, um trabalho maçante de memorização das duas condições. Não é que a representação por diagramas deva ser excluída das situações de ensino, mas focar o ensino de função numa representação tão estática, cujos conjuntos em causa raramente excedem três ou 
quatro elementos, pode gerar o "problema da trivialização" do conceito de função, problema sério porque fornece aos alunos uma imagem distorcida da Matemática (PONTE, 1990, p. 7).

Apresentar o conceito de função por meio de diagramas, que enfatizam a interpretação de função como uma relação binária, pode ser um grande contraexemplo de operacionalização da definição de função. Pois o professor pode apresentar uma definição que não vai usar. Sobre esse problema Lima afirma:

Funções são definidas por relações binárias, ponto de vista que nenhum matemático nem usuário da Matemática adota em seu dia-a-dia. Pior: esta generalidade inútil é rapidamente abandonada e todas as funções que surgem depois são bolinhas e flechinhas, ou então dadas por fórmulas (2001, p. 46).

Representação gráfica

Agora, representaremos graficamente a relação entre as variáveis, quantidade de pontinhos e posição, apresentadas no exemplo 1.

Gráfico 1 - Quantidade de pontinhos para as dez primeiras posições.

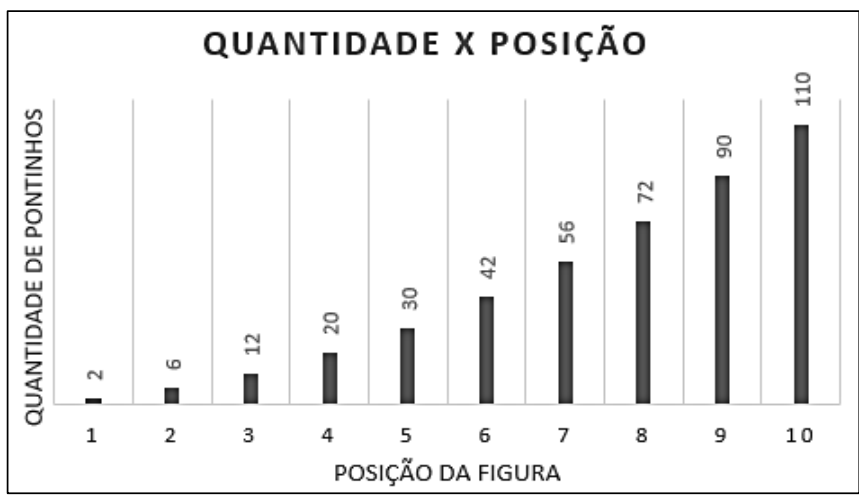

Fonte: Os autores.

O gráfico acima nos fornece uma base intuitiva fundamental, uma descrição visual de como as mudanças sofridas por uma grandeza $p$ provoca variações em outra $q$. Construir gráficos de funções constitui-se num processo de "visualização das leis de correspondência", o que facilita a análise de fenômenos, a descrição de regularidades e a interpretação de interdependências, pois além de ser outra forma de representar o conceito de função, o gráfico pode trazer informações adicionais.

Em geral, um gráfico é uma ferramenta que possui a importante função de sintetizar um conjunto de dados, facilitando a comunicação, análise e interpretação dos mesmos. A representação gráfica da relação funcional entre variáveis permite ao indivíduo vislumbrar o comportamento do fenômeno modelado, não é, pois, apenas um expediente de apelo visual, mas sim um instrumento cuja correta interpretação se constitui num processo de resolução de problemas.

Os gráficos se apresentam como uma ferramenta cultural que pode ampliar a capacidade humana de tratamento de informações quantitativas e de estabelecimento de relações entre as mesmas. A apresentação gráfica é frequentemente associada à coordenação de informações quantitativas dispostas em dois eixos perpendiculares; 
um horizontal (chamado eixo dos $\mathbf{x}$ ou abscissa) e um vertical (eixo dos $\mathbf{y}$ ou ordenada) (MONTEIRO, 1999, p. 1).

Embora existam outros tipos de gráficos que podem expressar o conceito de função, o tipo mais utilizado no ensino de funções é o gráfico cartesiano, isso porque as figuras no plano cartesiano podem ser descritas por fórmulas, o que possibilita a articulação entre duas formas de representar o conceito: a gráfica e a analítica. Considerando o contexto histórico, Caraça (1951) afirma que o conceito de função serviu como elemento definidor da unificação de dois campos que passaram aproximadamente dois mil anos separados: o campo analítico e o geométrico.

A representação gráfica, no ensino de funções, não deve ser posta em segundo plano ou ser vista como uma mera extensão de uma fórmula. O trabalho quase que exclusivo com as expressões analíticas que definem as funções pode passar a impressão de que uma função é apenas uma fórmula. Nesse sentido, as manipulações algébricas acabam sendo consideradas a única via para encontrar soluções de problemas sobre funções. Nesse contexto, Filho e Menezes (2010, p. 7) ao analisarem os dados de sua pesquisa discutem sobre:
a tendência dos alunos, mesmo nas questões inteiramente relacionadas à interpretação gráfica, de utilizarem estratégias e procedimentos com cálculos para resolverem os problemas. Especificamente, têm-se evidências de que os alunos concebem o cálculo como a única, ou mais relevante, via para encontrar soluções.

Há, ainda, a situação em que o aprendiz sabe definir corretamente o conceito de função, mas não sabe identificar quando um gráfico cartesiano representa uma função. Isso pode ocorrer quando uma única representação do conceito é enfatizada, geralmente as expressões analíticas, e as demais relegadas a atividades repetitivas. Isso pode dificultar a construção significativa do conceito, tendo em vista que um conceito transcende suas representações. Sem falar nas terminologias que são apresentadas quando uma representação é trabalhada, elas são apresentadas de forma desconexa, o que faz com que as representações do conceito de função: verbal, tabular, por diagramas, analítica e gráfica, se isolem.

No caso dos gráficos, existe uma regra para verificar se um gráfico cartesiano representa ou não o conceito de função. Essa regra pode ser assim apresentada: considerando o gráfico cartesiano da relação $f$ de $A$ em $B$, se a reta paralela ao eixo das ordenadas conduzida pelo ponto $(x, 0)$, em que $x \in A$, sempre intersectar o gráfico de $f$ em um único ponto (gráfico 3), então esse gráfico representa uma função. Se a paralela, nessas condições, intersectar o gráfico em mais de um ponto, o mesmo não representará uma relação funcional (gráfico 2). 
Gráfico 2 - Circunferência $x^{2}+y^{2}=1$.

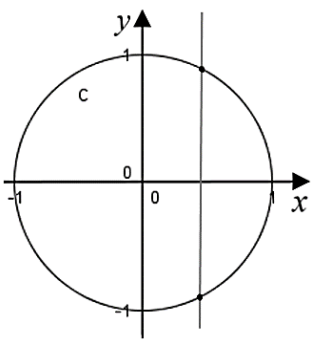

Fonte: Os autores.
Gráfico 3 - Função definida por $f(x)=1 / x \operatorname{com} x>0$.

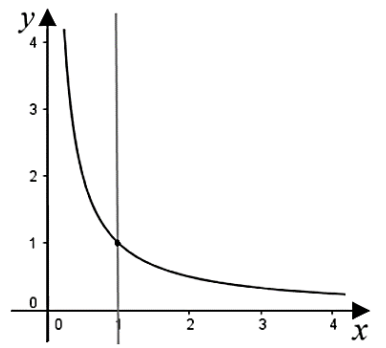

Fonte: Os autores.

Por que se reta paralela intersectar o gráfico em dois ou mais pontos, o mesmo não representará uma função?

Isso vem da própria definição de função e de como se constrói um gráfico cartesiano. Os gráficos cartesianos são formados por pontos $(x, y)$ que satisfazem condições específicas, por exemplo, numa circunferencia de raio 1, centrada na origem, o quadrado da abscissa acrescido do quadrado da ordenada deve ser igual a 1 , ou seja, os pontos satisfazem a condição $x^{2}+y^{2}=1$ (gráfico 2). O gráfico cartesiano de uma função é ainda mais específico, pois é exigido que cada abscissa esteja associada a uma única ordenada. Como as ordenadas $f(x)$, em termos gráficos, são as alturas dos pontos de abscissa $x$, então cada ponto deve ter, grosso modo, uma única altura. Essa regra não passa de um macete para se transferir para a linguagem gráfica a condição: sem ambiguidades, que a regra que define uma função deve obedecer, isso quer dizer: mais terminologias.

Em suma, nesta seção, destacamos a questão do acréscimo de terminologias novas toda vez em que uma representação é trabalhada, isto é, regras que são utilizadas para identificar, por exemplo, se determinados gráficos ou diagramas representam o conceito de função. Isso tem como consequência o isolamento dos registros de representação, pois tais regras são desconexas e restritas ao contexto de um único registro, o que inviabiliza a coordenação entre as várias representações do conceito de função. $\mathrm{O}$ grande entrave que o isolamento impinge, refere-se à própria apreensão do objeto matemático em jogo, pois, conforme Duval (2012), a apreensão de um objeto depende de que o aprendiz consiga realizar tratamentos em diferentes registros de representação e transitar de um para outro o mais espontaneamente possível.

Sobre a ideia do isolamento de registros de representação, Duval (2012, p. 283) afirma:

Naturalmente, a ausência de coordenação não impede toda compreensão. Mas esta compreensão, limitada ao contexto semiótico de um registro apenas, não favorece em nada as transferências e as aprendizagens ulteriores: torna os conhecimentos adquiridos pouco ou não utilizáveis em outras situações aonde deveriam realmente ser utilizados. Em definitivo, esta compreensão mono registro conduz a um trabalho às cegas, sem possibilidade de controle do "sentido" daquilo que é feito. 
A contínua exemplificação da operacionalização da definição de função, por parte do professor, pode subsidiar a superação do problema do isolamento de representações. Como foi discutido nesta seção, a definição apresentada pelo professor surge como uma mera formalidade, que é posteriormente abandonada. Adotando a constante exemplificação, o docente poderia guiar-se pela definição apresentada toda vez em que discutisse porque determinadas relações entre variáveis são funcionais e porque certas tabelas, gráficos, fórmulas e diagramas representam o conceito de função, o que colocaria a definição do conceito de função como um elo de ligação entre as demais representações, uma referência facilitadora da articulação entre essas representações. Como a concepção de função dos alunos está ligada à concepção de função de seus professores (PIRES; SILVA, 2015), temos um precioso momento para apresentar e utilizar uma definição de função que sirva para modelar aquela concepção inicial dos aprendizes, explicitando na prática docente uma forma mais significativa de trabalhar o conteúdo funções.

Além de utilizar a definição de função que adotou, o professor poderia proporcionar um ambiente rico em representações, trabalhando os contras e prós de cada uma delas, levando o aprendiz a entender que todas as representações são diferentes fontes de informações sobre o conceito de função, mas que o conceito em si não pode ser reduzido às suas representações.

Embora as representações sejam de suma importância, o que importa não são elas, mas sim os objetos matemáticos que são representados (o conteúdo). Os objetos matemáticos nunca devem ser confundidos com a forma, isto é, com as representações que se fazem deles. Nesse contexto, o recurso a uma pluralidade de representações, como aponta Duval (2012), é uma condição para a superação da confusão entre representante e representado. Para este autor, essa confusão acarreta

\begin{abstract}
uma perda de compreensão e os conhecimentos adquiridos tornam-se rapidamente inúteis ao longo de seu contexto de aprendizagem: seja por não lembrar ou porque permanecem como representações "inertes" que sugerem nenhum tratamento (DUVAL, 2012, p. 268, grifo nosso).
\end{abstract}

\title{
Considerações finais
}

Considerando a operacionalização da definição como o elemento desencadeador do processo de aquisição do conceito de função, objetivamos, neste artigo, tecer reflexões sobre como favorecer a operacionalização da definição de função por parte do aprendiz.

Com base na análise histórico-epistemológica do conceito de função, e na discussão acerca de suas múltiplas representações, acreditamos que para favorecer a operacionalização três aspectos devem ser considerados:

a) introduzir o conceito de função como uma relação entre quantidades variáveis para, posteriormente, defini-lo como relação entre conjuntos; 
b) apresentar o conceito de função dentro diferentes contextos;

c) enunciar uma definição de função que realmente seja utilizada.

Apresentar o conceito de função por meio de sua definição formal apoiada na representação por diagramas é esperar que o aluno apreenda em poucas aulas a forma mais geral de um conceito matemático que a humanidade levou séculos para formalizar. Introduzir o conceito de função por meio de relações binárias pode dificultar a construção de um campo de significados para o conceito. Como preconiza (a), devemos obedecer ao movimento histórico do desenvolvimento do conceito de função. Partir do entendimento da noção de variação, de dependência, e da percepção de regularidades, para o formalismo inerente à interpretação de função como relação entre conjuntos.

Trabalhar um conteúdo exclusivamente por meio da abordagem definição-exemploexercício pode obscurecer a análise do professor e, consequentemente, a aprendizagem de seus alunos. O docente poderia pensar que um aluno apreendeu um conceito e atribuiu significação ao mesmo, quando na verdade o contexto linear onde foi apresentado facilitou a memorização de toda terminologia relacionada a ele. Consoante (b), o docente poderia apresentar o conceito de função dentro de múltiplos contextos; contextos que requeiram máxima transformação do conhecimento adquirido. Assim, diferentes atividades devem aplicadas, não só em contextos livrescos, mas atividades concretas onde toda terminologia relacionada ao conceito surja como uma ferramenta prática para lidar com problemas com referência na realidade. Atividades baseadas em (b) devem, também, recorrer à pluralidade de representações do conceito de função, ou seja, a atividades em que os alunos tenham a oportunidade de trabalhar com tabelas, fórmulas e gráficos, para que possam entender os contras e prós de cada uma dessas representações e que essas representações são diferentes fontes de informação sobre um mesmo objeto.

Segundo (c), o professor deveria trabalhar com a definição de função que adotou, exemplificando permanentemente o processo de operacionalização da definição de função. Sempre que possível, o professor deve explicar, por meio da definição adotada, porque determinadas relações entre variáveis são funcionais e porque determinadas tabelas, digramas ou gráficos representam o conceito de função.

Em síntese, as três categorias apresentadas podem ser utilizadas em sequências didáticas que visem à iniciação dos alunos no tema função ou a modelar as concepções dos aprendizes já iniciados. Para entendermos a relevância da operacionalização da definição de função, devemos ter claro que articular as múltiplas representações do conceito de função é muito mais do que saber se determinada tabela, diagrama ou gráfico representa uma relação funcional, implica a construção semântica desse conceito. Portanto, no que se refere à aprendizagem significativa do conceito de função, devemos eleger uma definição operacional 
como o estopim de todo o processo, ou seja, deve-se operacionalizar para articular e articular para compreender.

\section{Referências}

ALVARENGA, K.; BARBOSA, C. V.; FERREIRA, G. M. O conceito de função: o desenvolvimento baseado em alguns modelos desde o ano de 2000 a. C. até o século XX. Revemat, v. 9, n. 1, p. 159-178, 2014.

ÁVILA, G. Evolução do conceito de função e integral. Revista Matemática Universitária, n. 1, p. 14-46, 1985.

BOTELHO, L.; REZENDE, W. Um breve histórico do conceito de função. Caderno Dá Licença, v. 6, p. 65-75, 2007.

BOYER, C. B. História da matemática. 2. ed. São Paulo: Edgard Blücher, 1996.

CARAÇA, B. J. Conceitos fundamentais da matemática. Lisboa: Tipografia Matemática, Ltda, 1951. Disponível em: <http://www.im.ufrj.br/nedir/disciplinasPagina/Caraca_ConceitosFundamentais.pdf > . Acesso em: 20 dez. 2014.

COSTA, A. C. Conhecimentos de estudantes universitários sobre o conceito de função. Dissertação de Mestrado em Educação Matemática - Pontifícia Universidade Católica de São Paulo, São Paulo, 2004.

DUVAL, R. Registros de representação semiótica e funcionamento cognitivo do pensamento. Revemat, v. 7, n. 2, p. 266-297, 2012.

FILHO, M. S. M.; MENEZES, J. E. Como os alunos do ensino médio estão construindo e interpretando gráficos de funções polinomiais $1^{\circ}$ e $2^{\circ}$ graus. In: ENCONTRO NACIONAL DE EDUCAÇÃO MATEMÁTICA - ENEM, 10, 2010, Salvador. Anais... Salvador, 2010.

FONSECA, V. G.; SANTOS, A. R.; NUNES, W. V. Estudo epistemológico do conceito de função: uma retrospectiva. In: ENCONTRO NACIONAL DE EDUCAÇÃO MATEMÁTICA - ENEM, 11, 2013, Curitiba. Anais... Curitiba, 2013.

GIL, A. C. Métodos e técnicas de pesquisa social. 6.ed. São Paulo: Atlas, 2008.

KLEINER, I. Evolution of the function concept: a brief survey. The College Mathematics Journal, Washington D.C., v. 20, n. 4, p. 282-300, 1989. Disponível em: <www.maa.org/pubs/Calc_ar ticles/ma001.pdf >. Acesso em: 15 ago. 2016.

LIMA, E. L.; CARVALHO, P. C. P.; WAGNER, E.; MORGADO, A. C. A Matemática do Ensino Médio. Coleção do Professor de Matemática. v. 1. 10.ed. Rio de Janeiro: SBM, 2012.

LIMA, T. C. S.; MIOTO, R. C. T. Procedimentos metodológicos na construção do conhecimento científico: a pesquisa bibliográfica. Revista Katálysis, v. 10, n. esp., p. 37-45, 2007.

MARCONI, M. A.; LAKATOS, E. M. Fundamentos de metodologia científica. 5.ed. São Paulo: Atlas 2003.

MONTEIRO, C. E. F. Interpretação de gráficos: atividade social e conteúdo de ensino. In: REUNIÃO ANUAL DA ASSOCIAÇÃO NACIONAL DE PÓS-GRADUAÇÃO E 
PESQUISA EM EDUCAÇÃO - ANPEd, 22, 1999, Caxambu. Anais... Caxambu, 1999. 1 CD-ROM.

PIRES, R. F.; SILVA, B. A. Função: concepções daquele que ensina e daquele que aprende. EM TEIA, v. 5, n. 3, p. 1-25, 2015.

PONTE, J. P. O conceito de função no currículo de matemática. Educação e Matemática, Portugal, n. 15, p. 3-9, 1990.

REZENDE, W. M. Uma análise histórica-epistêmica da operação de limite. Dissertação de Mestrado em Educação Matemática - Universidade Santa Úrsula, Rio de Janeiro, 1994.

RÜTHING, D. Some definitions of the concept of function from Joh. Bernoulli to N. Bourbaki. The Mathematical Intelligencer, v. 6, n. 4, p. 72-77, 1984.

SIERPINSKA, A. On understanding the notion of function. In: DUBINSKY, E.; HAREL, G. (Ed.). The concept of function: aspects of epistemology and pedagogy. M. A. A. Notes, Washington, D.C., v. 25, 1992. p. 25-58.

SILVA, M. H. M.; REZENDE, W. M. Análise histórica do conceito de função. Caderno Dá Licença, v. 2, p. 28-33, 1999.

VINNER, S. Concept definition, concept image and the notion of function. International Journal of Mathematical Education in Science and Technology, v. 14, n. 3, p. 293-305, 1983.

YOUSCHKEVICH, A. P. The concept of function up to the middle of the 19th century. Archive for History of Exact Sciences, v. 16, n. 1, p.37-85, 1976.

\section{SOBRE OS AUTORES}

JERSON SANDRO SANTOS DE SOUZA. É licenciado em Matemática pela Universidade Federal do Amazonas (2013) e mestrando em Ensino de Ciências e Matemática pela mesma instituição. Dedica-se ao estudo dos processos de ensino-aprendizagem em Ciências e Matemática. Atua como professor de Matemática do ensino básico.

LEANDRO DE OLIVEIRA SOUZA. Doutor em Ensino de Ciências e Matemática (2013), Mestre em Ensino de Ciências e Matemática pela Universidade Cruzeiro do Sul (2009), Pósgraduação em Ensino de Matemática pela Universidade Braz Cubas (2003), graduação em Pedagogia com hab. em administração e supervisão pela Universidade Guarulhos (2006) e graduação em Licenciatura em Matemática pela Universidade Braz Cubas (2001). Atualmente é professor da Universidade Federal de Uberlândia (UFU/FACIP), e orienta pelos programas de Pós-graduação em Ciências e Tecnologia para Recursos Amazônicos (UFAM) e de Pósgraduação em Ensino de Ciências e Matemática (UFAM). Participa do grupo de pesquisas GEPEE, produzindo pesquisas na área de ensino de probabilidade e estatística, tecnologia, educação estatística e formação de professores.

Recebido: 18 de fevereiro de 2017.

Revisado: 09 de maio de 2017.

Aceito: 01 de junho de 2017. 